PROCEEDINGS OF THE

AMERICAN MATHEMATICAL SOCIETY

Volume 135, Number 11, November 2007, Pages 3461-3464

S 0002-9939(07)08909-5

Article electronically published on July 2, 2007

\title{
A CHARACTERIZATION OF MODULES LOCALLY OF FINITE INJECTIVE DIMENSION
}

RYO TAKAHASHI

(Communicated by Bernd Ulrich)

\begin{abstract}
In this note, we characterize finite modules locally of finite injective dimension over commutative Noetherian rings in terms of vanishing of Ext modules.
\end{abstract}

\section{INTRODUCTION}

Let $R$ be a commutative Noetherian ring. Goto 2$]$ proved the following theorem.

Theorem 1.1 (Goto). The following are equivalent:

(1) $R$ is Gorenstein;

(2) For every finite $R$-module $M$, there exists an integer $n$ such that $\operatorname{Ext}_{R}^{i}(M, R)$ $=0$ for all $i>n$.

We should note that this theorem remains valid even in the case where the ring $R$ has infinite Krull dimension.

The purpose of this note is to give a characterization of finite modules locally of finite injective dimension. Our theorem is the following.

Theorem 1.2. The following are equivalent for a finite $R$-module $N$ :

(1) $\operatorname{id}_{R_{\mathfrak{p}}} N_{\mathfrak{p}}<\infty$ for every $\mathfrak{p} \in \operatorname{Spec} R$;

(2) For every finite $R$-module $M$, there exists an integer $n$ such that $\operatorname{Ext}_{R}^{i}(M, N)$ $=0$ for all $i>n$.

This theorem is a generalization of Goto's. In fact, applying our theorem to $N=R$, we immediately obtain Goto's theorem.

\section{Proof of the theorem}

We denote by $\operatorname{CM}(R)$ the Cohen-Macaulay locus of $R$, that is, the set of prime ideals $\mathfrak{p}$ of $R$ such that the local ring $R_{\mathfrak{p}}$ is Cohen-Macaulay. The following lemma can be shown in a similar way to the proof of [3, Theorem 24.5].

Lemma 2.1. Let $\mathfrak{p}$ be a prime ideal of $R$ such that both $R_{\mathfrak{p}}$ and $R / \mathfrak{p}$ are CohenMacaulay rings. Then there exists an element $f \in R-\mathfrak{p}$ such that $D(f) \cap V(\mathfrak{p}) \subseteq$ $\mathrm{CM}(R)$.

Received by the editors March 13, 2006 and, in revised form, August 19, 2006.

2000 Mathematics Subject Classification. Primary 13D05, 13D07.

Key words and phrases. Injective dimension, Cohen-Macaulay locus.

(C)2007 American Mathematical Society 
It is known that the Cohen-Macaulay locus of a homomorphic image of a CohenMacaulay ring is an open subset; see [3, Exercises 24.2]. To prove our theorem, we need to generalize this fact. For an ideal $I$ of $R$, let $\operatorname{CM}_{R}(R / I)$ denote the set of prime ideals $\mathfrak{p} \in V(I)$ such that the local ring $(R / I)_{\mathfrak{p}}$ is Cohen-Macaulay.

Lemma 2.2. Let $I$ and $J$ be ideals of $R$. Suppose that $V(J)$ is contained in $\mathrm{CM}(R)$. Then the following hold:

(1) For any prime ideal $\mathfrak{p} \in \mathrm{CM}_{R}(R / I) \cap V(J)$, there exists an element $f \in R-\mathfrak{p}$ such that $D(f) \cap V(\mathfrak{p}) \subseteq \mathrm{CM}_{R}(R / I)$.

(2) There exists an ideal $K$ of $R$ such that

$$
\mathrm{CM}_{R}(R / I) \cap V(J)=D(K) \cap V(I) \cap V(J) .
$$

In other words, $\mathrm{CM}_{R}(R / I) \cap V(J)$ is an open subset of $V(I) \cap V(J)$ in the relative topology induced by the Zariski topology of $\operatorname{Spec} R$.

Proof. (1) Let $\mathfrak{p} \in \mathrm{CM}_{R}(R / I) \cap V(J)$. Then by the assumption that $V(J)$ is contained in $\mathrm{CM}(R)$, the ring $R_{\mathfrak{p}}$ is a Cohen-Macaulay local ring. Making a similar argument to the proof of [3, Theorem 24.5], we can assume without loss of generality that there is an $R$-regular sequence $\boldsymbol{x}=x_{1}, x_{2}, \ldots, x_{n}$ in $\mathfrak{p}$ with $\mathfrak{p}^{r} \subseteq \boldsymbol{x} R$ for some $r>0$ and that $\overline{\mathfrak{p}}^{i} / \overline{\mathfrak{p}}^{i+1}$ is a free $\bar{R} / \overline{\mathfrak{p}}$-module for all $i>0$, where $\bar{R}=R / \boldsymbol{x} R$ and $\overline{\mathfrak{p}}=\mathfrak{p} / \boldsymbol{x} R$.

We have only to prove that the residue ring $R / \mathfrak{p}$ is Cohen-Macaulay. In fact, if $R / \mathfrak{p}$ is Cohen-Macaulay, then so are $(R / I) /(\mathfrak{p} / I)$ and $(R / I)_{\mathfrak{p} / I}$ since $\mathfrak{p}$ is in $\mathrm{CM}_{R}(R / I)$. Hence Lemma 2.1 implies that there is an element $f \in R-\mathfrak{p}$ such that $D(\bar{f}) \cap V(\mathfrak{p} / I)$ is contained in $\operatorname{CM}(R / I)$, where $\bar{f}$ denotes the residue class of $f$ in $R / I$. We easily see that $D(f) \cap V(\mathfrak{p})$ is contained in $\mathrm{CM}_{R}(R / I)$.

Let us show that $R / \mathfrak{p}$ is a Cohen-Macaulay ring. It is easy to see from 3 , Exercises 24.1] that $R / \mathfrak{p}=\bar{R} / \overline{\mathfrak{p}}$ is Cohen-Macaulay if and only if so is $\bar{R}$. Take a prime ideal $\mathfrak{q} \in V(\boldsymbol{x})=V(\mathfrak{p})$. Then we have $\mathfrak{q} \supseteq \mathfrak{p} \supseteq J$; hence $\mathfrak{q} \in V(J) \subseteq \mathrm{CM}(R)$. Therefore $R_{\mathfrak{q}}$ is a Cohen-Macaulay local ring, and so is $\bar{R}_{\mathfrak{q}}$, because $\boldsymbol{x}$ is an $R_{\mathfrak{q}^{-}}$ regular sequence. This shows that $\bar{R}$ is a Cohen-Macaulay ring. Thus we conclude that the residue $\operatorname{ring} R / \mathfrak{p}$ is Cohen-Macaulay, as desired.

(2) Set $U=\left\{\mathfrak{p} / I+J \mid \mathfrak{p} \in \mathrm{CM}_{R}(R / I) \cap V(J)\right\}$. This is a subset of Spec $R / I+J$. Note that this subset is stable under generalization. Let $P \in U$. Then there is a prime ideal $\mathfrak{p} \in \mathrm{CM}_{R}(R / I) \cap V(J)$ such that $P=\mathfrak{p} / I+J$. By the assertion (1) of the lemma, the set $D(f) \cap V(\mathfrak{p})$ is contained in $\mathrm{CM}_{R}(R / I)$ for some $f \in R-\mathfrak{p}$. Denote by $\bar{f}$ the residue class of $f$ in $R / I$. It is easy to see that $P \in D(\bar{f}) \cap V(P) \subseteq U$. Thus $U$ contains a nonempty open subset of $V(P)$. By virtue of the topological Nagata criterion [3, Theorem 24.2], $U$ is an open subset of $\operatorname{Spec} R / I+J$; we have $U=D(K / I+J)$ for some ideal $K$ of $R$ containing $I+J$. Then it is easily checked that $\mathrm{CM}_{R}(R / I) \cap V(J)=D(K) \cap V(I) \cap V(J)$.

Now, we can prove our theorem.

Proof of Theorem 1.2. $(2) \Rightarrow(1)$ : Let $\mathfrak{p}$ be a prime ideal of $R$. Then there is an integer $n$ such that $\operatorname{Ext}_{R}^{i}(R / \mathfrak{p}, N)=0$ for all $i>n$. Hence we have $\operatorname{Ext}_{R_{\mathfrak{p}}}^{i}\left(\kappa(\mathfrak{p}), N_{\mathfrak{p}}\right)=0$ for all $i>n$. Therefore by [1, Theorem 3.1.14] we obtain $\operatorname{id}_{R_{\mathfrak{p}}} N_{\mathfrak{p}} \leq n<\infty$.

$(1) \Rightarrow(2)$ : First of all, note that (2) is equivalent to the statement that for each ideal $I$ of $R$ there is an integer $n$ such that $\operatorname{Ext}_{R}^{i}(R / I, N)=0$ for all $i>n$. (This can easily be proved by induction on the number of generators of the $R$-module 
$M$.) Suppose that there exists an ideal $I$ of $R$ such that for any integer $n$ there is an integer $i>n$ such that $\operatorname{Ext}_{R}^{i}(R / I, N) \neq 0$. We want to derive a contradiction. Since $R$ is Noetherian, one can choose $I$ to be a maximal one among such ideals. Making a similar argument to the proof of Theorem [1.1, we see that the ideal $I$ is prime and that for any element $f \in R-I$, the map

$$
\operatorname{Ext}_{R}^{i}(R / I, N) \stackrel{f}{\rightarrow} \operatorname{Ext}_{R}^{i}(R / I, N)
$$

is an isomorphism for $i \gg 0$.

Claim 1. One has $I \in \operatorname{Supp}_{R} N \subseteq \operatorname{CM}(R)$.

Proof of Claim. Our assumption (1) implies that for any $\mathfrak{p} \in \operatorname{Supp}_{R} N$, the nonzero finite $R_{\mathfrak{p}}$-module $N_{\mathfrak{p}}$ has finite injective dimension. Hence $R_{\mathfrak{p}}$ is a Cohen-Macaulay local ring; see [1, Corollary 9.6.2 and Remarks 9.6.4]. Thus $\operatorname{Supp}_{R} N$ is contained in $\operatorname{CM}(R)$. On the other hand, assume that $I$ is not in $\operatorname{Supp}_{R} N$. Then there exists an element $f \in \operatorname{Ann}_{R} N-I$, and the map $\operatorname{Ext}_{R}^{i}(R / I, N) \stackrel{f}{\rightarrow} \operatorname{Ext}_{R}^{i}(R / I, N)$ is an isomorphism for $i \gg 0$. Since $f N=0$, this map is the zero map, and we get $\operatorname{Ext}_{R}^{i}(R / I, N)=0$ for $i \gg 0$. It follows from this contradiction that $I$ belongs to $\operatorname{Supp}_{R} N$.

Noting that $\operatorname{Supp}_{R} N=V\left(\operatorname{Ann}_{R} N\right)$, we see from Claim 1 and Lemma 2.2(2) that there is an ideal $K$ of $R$ such that $\mathrm{CM}_{R}(R / I) \cap \operatorname{Supp}_{R} N=D(K) \cap V(I) \cap$ $\operatorname{Supp}_{R} N$. The localization $(R / I)_{I}=\kappa(I)$ is a field, hence a Cohen-Macaulay ring. It is seen from Claim 1 again that $I \in \mathrm{CM}_{R}(R / I) \cap \operatorname{Supp}_{R} N \subseteq D(K)$. Thus there is an element $f \in K-I$.

Claim 2. For any prime ideal $\mathfrak{p} \in D(f)$ and any integer $i>$ ht $I$, one has $\operatorname{Ext}_{R_{\mathfrak{p}}}^{i}\left(R_{\mathfrak{p}} / I R_{\mathfrak{p}}, N_{\mathfrak{p}}\right)=0$.

Proof of Claim. We may assume that $\mathfrak{p}$ belongs to both $V(I)$ and $\operatorname{Supp}_{R} N$, because otherwise the module $\operatorname{Ext}_{R_{\mathfrak{p}}}^{i}\left(R_{\mathfrak{p}} / I R_{\mathfrak{p}}, N_{\mathfrak{p}}\right)$ automatically vanishes. Hence Claim 1 implies that $\mathfrak{p}$ belongs to $\mathrm{CM}(R)$, namely the local ring $R_{\mathfrak{p}}$ is Cohen-Macaulay. Added to it, since $D(f)$ is contained in $D(K)$, we have $\mathfrak{p} \in D(K) \cap V(I) \cap \operatorname{Supp}_{R} N \subseteq$ $\mathrm{CM}_{R}(R / I)$, and therefore $R_{\mathfrak{p}} / I R_{\mathfrak{p}}$ is Cohen-Macaulay. Thus we get the following equalities:

$$
\operatorname{depth} R_{\mathfrak{p}}-\operatorname{depth} R_{\mathfrak{p}} / I R_{\mathfrak{p}}=\operatorname{dim} R_{\mathfrak{p}}-\operatorname{dim} R_{\mathfrak{p}} / I R_{\mathfrak{p}}=\text { ht } I R_{\mathfrak{p}}=\text { ht } I .
$$

Since $N_{\mathfrak{p}}$ is a finite $R_{\mathfrak{p}}$-module of finite injective dimension by assumption, it follows from the result of Ischebeck [1, Exercises 3.1.24] that $\operatorname{Ext}_{R_{\mathfrak{p}}}^{i}\left(R_{\mathfrak{p}} / I R_{\mathfrak{p}}, N_{\mathfrak{p}}\right)=0$ for every $i>$ ht $I$.

Claim 2 means that $\left(\operatorname{Ext}_{R_{f}}^{i}\left(R_{f} / I R_{f}, N_{f}\right)\right)_{\mathfrak{P}}=0$ for every $\mathfrak{P} \in \operatorname{Spec} R_{f}$ and every $i>$ ht $I$. Therefore, $\operatorname{Ext}_{R_{f}}^{i}\left(R_{f} / I R_{f}, N_{f}\right)=0$ for $i>$ ht $I$. The $R$-module $\operatorname{Ext}_{R}^{i}(R / I, N)$ is isomorphic to $\operatorname{Ext}_{R_{f}}^{i}\left(R_{f} / I R_{f}, N_{f}\right)$ for $i \gg 0$, and thus $\operatorname{Ext}_{R}^{i}(R / I, N)=0$ for $i \gg 0$. This contradiction completes the proof of our theorem.

\section{REFERENCES}

[1] Bruns, W.; Herzog, J. Cohen-Macaulay rings, revised edition. Cambridge Studies in Advanced Mathematics, 39. Cambridge University Press, Cambridge, 1998. MR 1251956 (95h:13020) 
[2] Goto, S. Vanishing of $\operatorname{Ext}_{A}^{i}(M, A)$. J. Math. Kyoto Univ. 22 (1982/83), no. 3, 481-484. MR674605 (84c:13019)

[3] Matsumura, H. Commutative ring theory. Translated from the Japanese by M. Reid. Cambridge Studies in Advanced Mathematics, 8. Cambridge University Press, Cambridge, 1986. MR879273 (88h:13001)

Department of Mathematics, School of Science and Technology, Meiji University, 1-1-1 Higashimita, Tama-Ku, KaWASAKi 214-8571, JaPAN

E-mail address: takahasi@math.meiji.ac.jp 